\title{
Bridgeless PFC single ended primary inductance converter in continuous current mode
}

Nor Akmal Rai, Mohd Junaidi Abdul Aziz, Mohd Rodhi Sahid, Mohd Rodhi Sahid

School of Electrical Engineering, Faculty of Engineering, Universiti Teknologi Malaysia, Malaysia

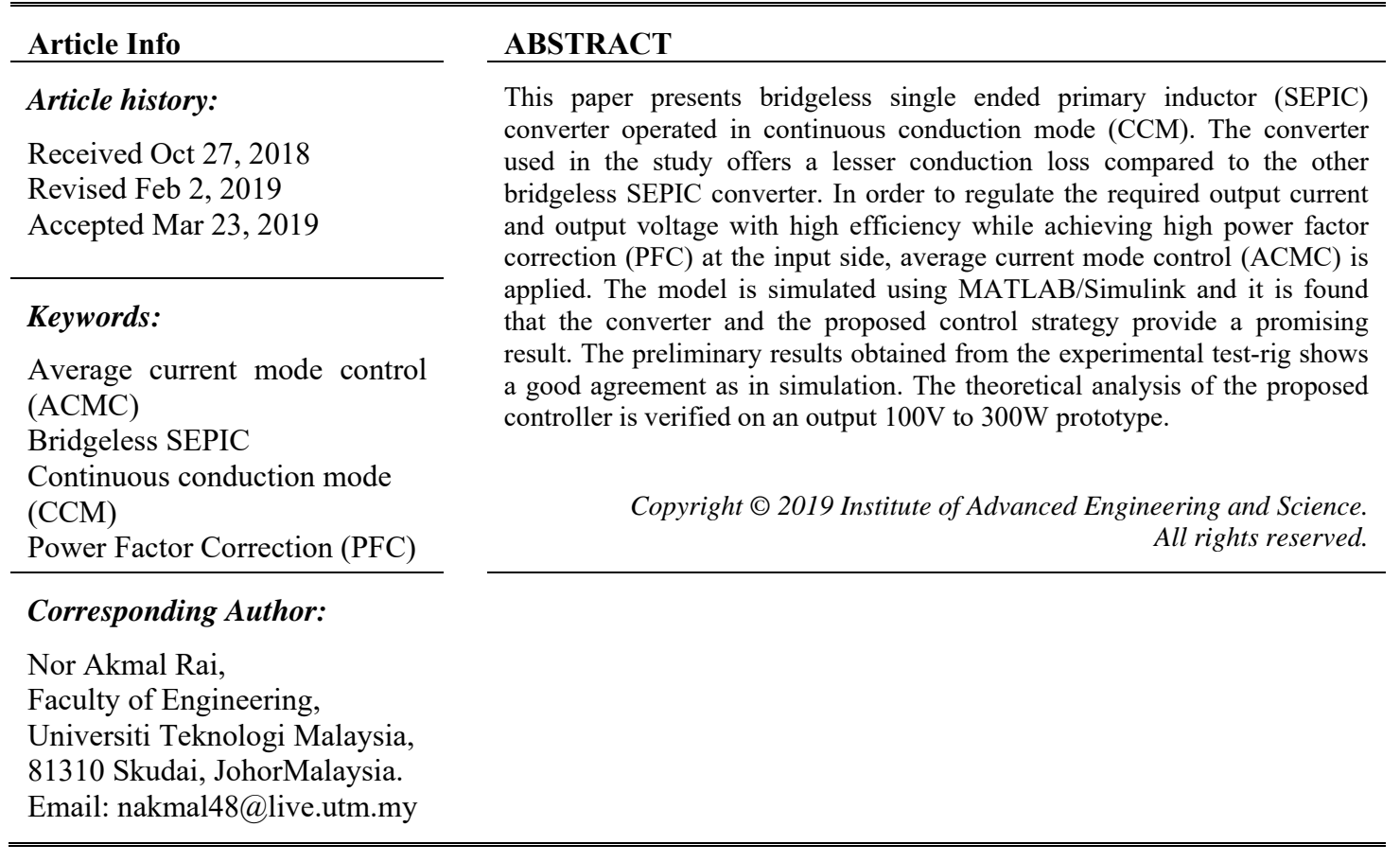

\section{INTRODUCTION}

A nonlinear load such as a battery, electronic device, and generator produce high harmonic distortion to ac input supply. This leads to losses in the supply and low power factor in the electrical system. PFC improves the power factor of electronic circuit. PFC ensure both input voltage and current are in phase, which leads to high power factor and reduces harmonic in supply. Normally, a conventional PFC can be accomplished by using a full bridge diode rectifier and dc-dc converter. Even though bridge rectifier has the ability to produce dc output but its drawback which produce an absolute sinusoidal voltage with high ripple and current that are highly nonsinusoidal [1]. A dc voltage produced by the rectifier is quite large and need to be regulated to a required value. Intended to this reason, a dc to dc converter are used to regulate an actual dc voltage with actual current waveform shape and low output ripple [2]. Moreover, this technique enhanced to PFC with low harmonic distortion [3].

There are various of dc-dc converter topologies used in PFC circuit. SEPIC converter is popular due to its advantages over another dc to dc converter. This converter produces an output voltage that is less or more than the input voltage, but with no polarity reversal [1, 4]. However, SEPIC converter is a 4th order converter since it has 4 storage elements in converter make it seldom uses due to difficulty in design the controller [5]. Nevertheless, this converter offers a surplus advantage compared to 2nd order converter where a lower input current ripple is possible to achieve $[5,6]$.

Literature studies state that a normal bridge SEPIC converter rectifier produces high conduction loss at input bridge diode hence reduces overall efficiency of the converter. This is due to a bridge rectifier consists of 4 diodes that produce high conduction loss during operation. It is possible to eliminate this high conduction loss with bridgeless converter. The efficiency of these converters is improved by removing the input bridge diode of the conventional bridge SEPIC converter. In the bridgeless converter number of 
elements conduct during each cycle are reduce as compare to the bridge rectifier. This significantly reduces losses in the circuit as reported in [7-14]. The Bridgeless SEPIC converter offer reduction of cost, light, increases efficiency at the same time maintaining near unity power factor performance. It is possible for the converter to operate in CCM and discontinuous conduction mode (DCM) depends on its application.

In CCM, the inductor current is always positive while for DCM inductor current is characterized by current returning to zero during every period. DCM causes large voltage stress, consequently gives impact on electromagnetic interference (EMI) into line [5]. Furthermore, at high power application, current stress and voltage stress in DCM become too large which affecting the efficiency of the converter [5, 15]. Hence, DCM normally used for low power application usually less than $200 \mathrm{~W}$ while CCM popularly used for medium and high power application [16-18]. This is due to CCM has lower conducted noise, lower conduction losses in the semiconductors and inductor, and lower inductor core loss [8, 18]. Additionally, it has low output voltage ripple. However, the design of CCM controller is more complex as compared to DCM. DCM has properties of self PFC since its capability to give higher power factor by the nature of their topologies [19, 20]. Hence, DCM has simple control and can achieve PFC by using simple control system. CCM required complex control system and required closed loop control to achieve PFC.

Current mode control typically work for converter operate in CCM. Among all current control mode, ACMC offers several advantages such as the ability to sense and control average inductor current while offering immunity to noise [21]. In PFC application, another significant feature of ACMC near the zero crossing of the line voltage, the converter operates with the maximum duty cycle. As a result, the dead angle period which encounter in peak current mode control is greatly reduced [22, 23]. Most of PFC applications has been widely adopte AMMC as a control technique for CCM converters [24, 25].

Bridgeless SEPIC converter in [7-12] focus at low power application operated in DCM using voltage control. This controller design is simple since all zero and pole are located at left hand plane make a tuning process easier. However, this controller provides high current and voltage stress for medium power application which cause some power loss to converter.

In this paper, CCM with ACMC applied to bridgeless SEPIC converter proposed in [9, 10] are studied. This paper is organized as follows. The bridgeless SEPIC converter detail circuit operation with average current controller are discussed in Section 2. The proposed circuit parameter, simulation result and preliminary result of hardware prototype are presents in Section 3. Finally, the conclusion is present in Section 4.

\section{RESEARCH METHOD}

\subsection{Circuit operation}

Bridgeless SEPIC converter as proposed in $[9,10]$ as in Figure 1, are simulated in CCM with an average current mode controller. The circuit consist of three inductors, three capacitors, two diode and two MOSFET apart from a resistor as its load.

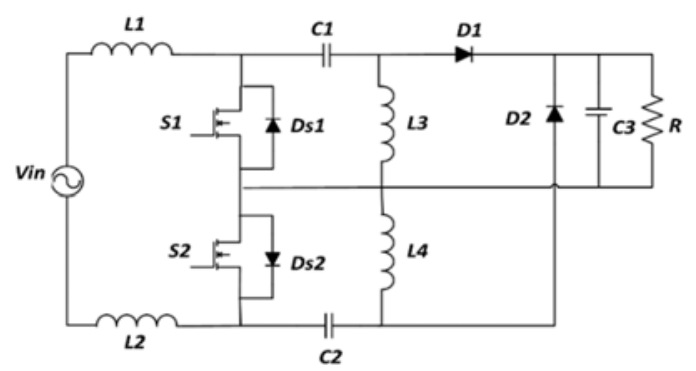

Figure 1. Bridgeless SEPIC circuit

This circuit has the same operation in both cycles, where each switch will only turn on in positive or negative half cycle. During the positive half cycle, only nine elements conduct which is L1, L2, S1, Ds2, C1, L3, D1, C3 and R as shown in Figure2. In negative half cycle L1, L2, S2, Ds1, C2, L3, D2, C3 and R are conduct as shown in Figure 3. This bridgeless SEPIC converter reduced number of component conduction during half cycle. At each half cycle of bridgeless SEPIC converters, it operates as basic dc-dc SEPIC converter.

Int J Pow Elec \& Dri Syst, Vol. 10, No. 3, Sep 2019 : 1427 - 1436 


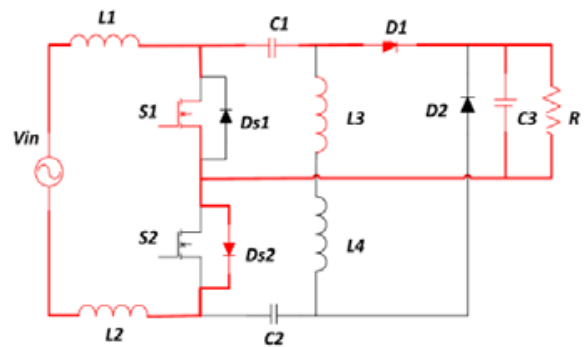

(a)

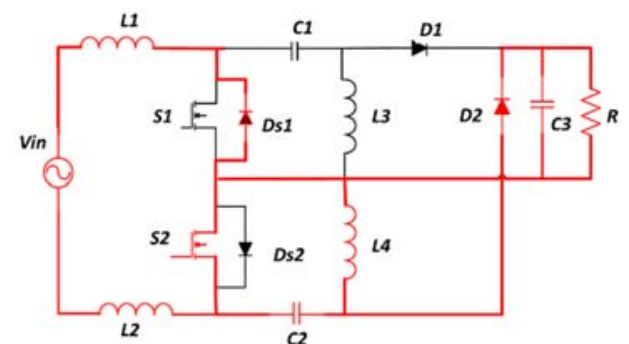

(b)

Figure 2. Operation of the bridgeless SEPIC converter in: (a) positive half cycle (b) negative half cycle

Bridgeless SEPIC operate in CCM and it consists of two mode operation per cycle. For positive half cycle it is operated in mode 1 and mode 2 as shown in Figure 3. The waveform of voltage and current operation for bridgeless SEPIC converter are shown in Figure 4.

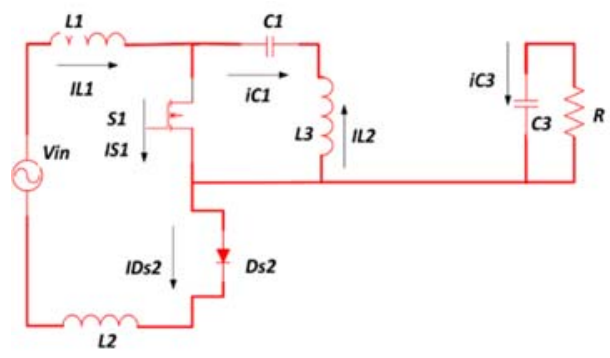

(a)

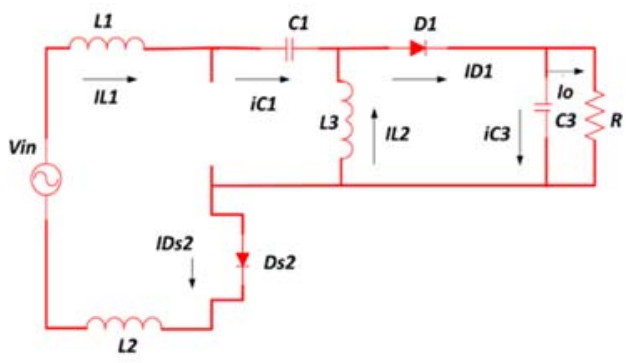

(b)

Figure 3. Positive half cycle operation in (a) mode 1 (b) mode 2

In mode 1, S1 and Ds2 are operate, current will flow through S1 and Ds2. L1 and L2 charge and are increase linearly to its peak depends on its duty cycle. In mode 2, S1 will turn off and D1 turn on, which allow current through it. Current across L1 decrease linearly due to discharging process through the C1, C3 and load. Since the circuit operation is symmetrical, the modes of operation for the negative half cycle are not shown here. Symmetrical feature of the converter in CCM is discussed in detail in $[9,10]$.

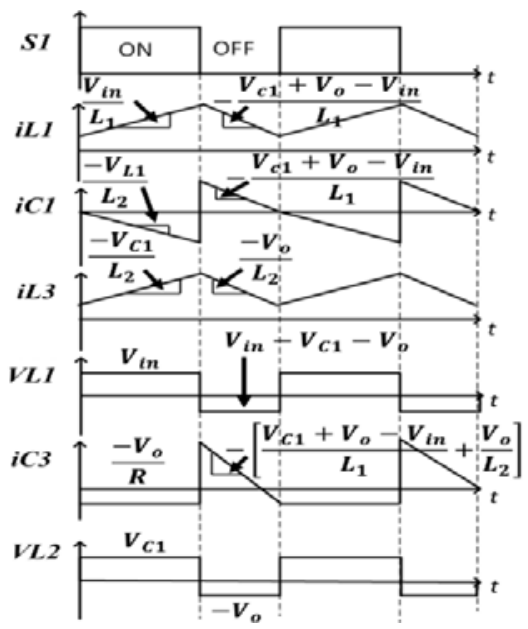

Figure 4. Waveform of current and voltages

Bridgeless PFC single ended primary inductance converter in continuous current mode (Nor Akmal Rai) 
In mode 1, S1 and Ds2 are operate, current will flow through S1 and Ds2. L1 and L2 charge and are increase linearly to its peak depends on its duty cycle

The voltage across inductor, L1

$$
L_{1} \frac{d i L_{1}}{d t}=\operatorname{Vin}
$$

The voltage across inductor, L2

$$
L 2 \frac{d i L_{2}}{d t}=V_{C 1}
$$

The current across series capacitor, $\mathrm{C} 1$

$$
C_{1} \frac{d i V_{C 1}}{d t}=-i_{L 2}
$$

The current across output capacitor, C3

$$
C_{3} \frac{d V_{C 3}}{d t}=-\frac{V_{o}}{R}
$$

In mode 2, S1 will turn off and D1 turn on, which allow current through it. Current across L1 decrease linearly due to discharging process through the C1, C3 and load. Since the circuit operation is symmetrical, the modes of operation for the negative half cycle are similar as in positive cycle but in opposite direction.

The voltage across inductor, L1

$$
L_{1} \frac{d i L_{1}}{d t}=V_{i n}-V_{C 1}-V_{o}
$$

The voltage across inductor, L2

$$
L_{2} \frac{d i L_{2}}{d t}=-V_{C 3}
$$

The current across series capacitor, $\mathrm{C} 1$

$$
C_{1} \frac{d V_{C 1}}{d t}=i_{L 1}
$$

The current across output capacitor, C3

$$
C_{3} \frac{d V_{C 3}}{d t}=i_{L 1}+i_{L 2}-\frac{V_{O}}{R}
$$

\subsection{Mathematical model}

In order to design the controller, it is mandatory to obtain the transfer function based on mathematical model to simplified controller tuning. Based on mode of operation of bridgeless SEPIC converter in CCM, the state space averaging modelling technique [25-27], are applied based on KCL and $\mathrm{KVL}$ of circuit during turn $\mathrm{ON}$ and turn OFF state as in equation (1-8).

Steady state dc model:

$0=A X+B U$

$Y=C X$

AC small signal model:

$$
\check{x}=A \tilde{x}+B \tilde{u}+B_{d} \tilde{d}
$$




$$
\hat{y}=C \tilde{x}
$$

$\tilde{x}$ - Vector of state variable

$\hat{y}$ - Vector of output system $\left(\mathrm{I}_{L 1}, V_{o}\right)$

A -State vector $\left(\widetilde{l L_{1}}, \widetilde{\imath L_{2}}, \widetilde{C_{1}}, \widetilde{V_{O}}\right)$

$\mathrm{B}$ - Input matrix

C - Matrix which connect output to the state variable

$\tilde{u}$ - Input variable $\left(V_{i n}\right)$

$B_{d} . \tilde{d}$ - Duty ratio variation for CCM [27]

The average matrices for steady state and liner small signal state space equation of bridgeless SEPIC converter based on (9) and (10) obtain as bellow:

$$
\begin{aligned}
& \frac{d}{d t}\left[\begin{array}{l}
\overrightarrow{l L_{1}} \\
\widetilde{l L_{2}} \\
\widetilde{C_{1}} \\
\widetilde{V_{O}}
\end{array}\right]=A \cdot\left[\begin{array}{l}
\widetilde{l L_{1}} \\
\widetilde{l L_{2}} \\
\widetilde{C_{1}} \\
\widetilde{V_{O}}
\end{array}\right]+B \cdot \tilde{d}+B_{d} \cdot V_{i n} \\
& \frac{d}{d t}\left[\begin{array}{l}
\widetilde{L L_{1}} \\
V_{O}
\end{array}\right]=C \cdot\left[\begin{array}{l}
\widetilde{l L_{1}} \\
\widetilde{l L_{2}} \\
\widetilde{C_{1}} \\
\widetilde{V_{O}}
\end{array}\right]
\end{aligned}
$$

Where:

$$
A=\left[\begin{array}{cccc}
0 & 0 & -\frac{1-D}{L_{1}} & -\frac{1-D}{L_{1}} \\
0 & 0 & \frac{D}{L_{2}} & -\frac{1-D}{L_{2}} \\
\frac{1-D}{C_{1}} & \frac{D}{C_{1}} & 0 & 0 \\
\frac{1-D}{C_{3}} & \frac{1-D}{C_{3}} & 0 & -\frac{1}{R C_{3}}
\end{array}\right], \mathrm{B}=\left[\begin{array}{c}
\frac{V_{O}}{L_{1} D} \\
\frac{V_{O}}{L_{2} D} \\
-\frac{V_{O}}{R C_{3}(1-D)} \\
-\frac{V_{O}}{R C_{3}(1-D}
\end{array}\right], B_{d}=\left[\begin{array}{c}
\frac{1}{L_{1}} \\
0 \\
0 \\
0
\end{array}\right], \mathrm{C}=\left[\begin{array}{llll}
1 & 0 & 0 & 0 \\
0 & 0 & 0 & 1
\end{array}\right]
$$

(11) and (12) consists of the ac perturbation. By using Laplace transformation in (11) and (12), yield a form of transfer function of bridgeless SEPIC converter.

$$
Y(s)=\left[\begin{array}{c}
I_{L 1}(s) \\
V_{o}(s)
\end{array}\right]=C\left(s I_{4}-A\right)^{-1} B_{d} \cdot D(s)+C\left(s I_{4}-A\right)^{-1} \cdot B V_{\text {in }}
$$

Where $\boldsymbol{I}_{\mathbf{4}}$ is a unity matrix, based on expansion and solving of equation 13 , the transfer function of inner loop and outer loop are:-

$$
\begin{aligned}
& G_{i d}=\frac{I_{L 1}(s)}{D(s)}=\frac{a a\left(s^{3}+a_{1} s^{2}+a_{2} s+a_{3}\right)}{\left.s^{4}+b_{1} s^{3}+b_{2} s^{2}+b_{3} s+b_{4}\right)} \\
& G_{v i}=\frac{V_{o}(s)}{I_{L 1}(s)}=\frac{-a b\left(s^{3}-a_{4} s^{2}+a_{5} s-a_{6}\right)}{s^{3}+a_{1} s^{2}+a_{2} s+a_{3}}
\end{aligned}
$$

With:

$$
\begin{aligned}
& a_{1}=\frac{1}{R C_{2}}\left[D\left(\frac{1+C_{3}}{C_{1}}\right)+1\right], a_{2}=\frac{D}{L_{2} C_{1}}\left[1+\left(\frac{L_{2}}{C_{3} R^{2}}\right)\right], a_{3}=2 D\left[R L_{1} C_{3} C_{1}\right], a_{4}=(1-D)^{2} \frac{R}{L_{1} D}\left[1+\frac{L_{1}}{L_{2}}\right], a_{5}=\frac{D}{L_{2} C_{1}} \\
& a_{6}=\frac{R(1-D)^{2}}{D L_{1} L_{2} C_{1}}, b_{1}=\frac{1}{R C_{2}}, b_{2}=\frac{1}{L_{1} C_{1}}\left[\left(\frac{L_{1}}{L_{2}} D^{2}\left(\frac{C_{1}}{C_{3}}\right)(1-D)^{2}\right)+\left((1-D)^{2} \frac{C_{1}}{C_{3}}\right)\right], b_{4}=\frac{(1-D)^{2}}{L_{1} L_{2} C_{1} C_{3}} \\
& a a=\frac{v_{O}}{L_{1} D}, a b=-\frac{L_{1} D}{R C_{3}(1-D)}
\end{aligned}
$$

Bridgeless PFC single ended primary inductance converter in continuous current mode (Nor Akmal Rai) 


\subsection{Control method}

Average current mode control is employed to control the bridgeless SEPIC converter operated in CCM. In $[9,10]$ the converter is operated in DCM using voltage control and suitable to be used at lower power application. The proposed controller for the converter mainly focus on medium power application is illustrated in Figure 5. Average current mode controller consists of two loops (cascade). Inner loop is for current controller while outer loop is for voltage controller.

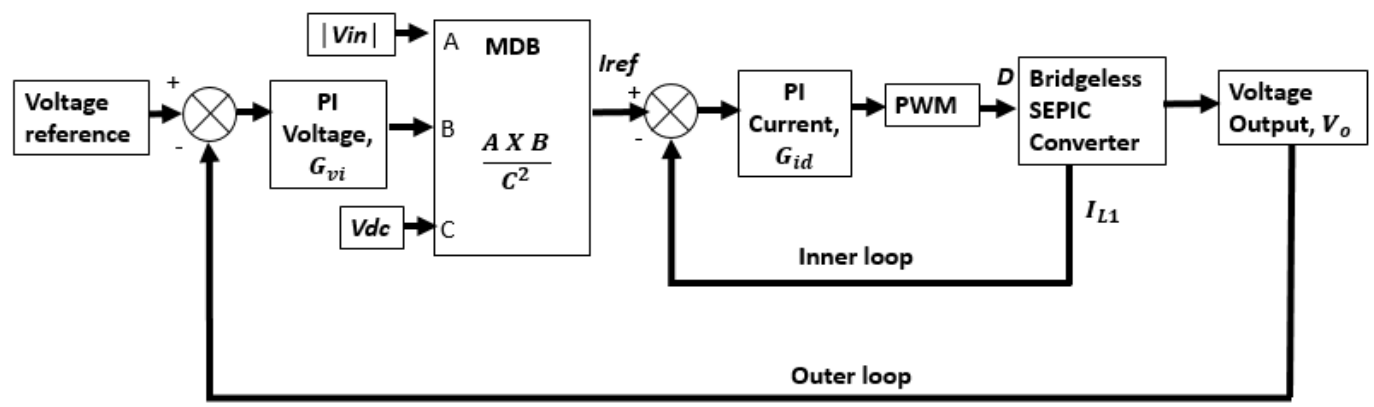

Figure 5. Average current mode control for bridgeless SEPIC using PID controller

In outer loop, the reference and actual output voltage of converter are compared, and the error is fed to generate the inductor current reference for inner loop. Outer loop also regulate voltage and to maintain it preferred set point. Meanwhile for inner loop the main purposed is to ensure the wave shaping inductor current with lead to improve input power factor. In inner loop, the actual inductor current is compared with the reference current produced by outer voltage. Any changes in inductor current, the current loop compensator will alter the duty ratio to ensure the output voltage remains as its desired value [28]. Output from inner loop is compared with saw tooth carrier to generated fixed frequency pulse width modulation (PWM) signal.

Since the converter operates in CCM, multiplication and dividing signal (MDB) are used to obtain the reference current input. It is an easy way to obtain high power factor by using this method since the reference current waveform is proportional to the input voltage waveform based on multiplier technique [24, 29]. MDB consist of multiplier and dividing of voltage input rectified, | Vin |, PI voltage error and average component of input rectified voltage, Vdc.

The controller is design using SISO tool in MATLAB/Simulink to obtain desired PI controller for inner and outer loop. By using transfer function as in section 2.2, the inner loop bandwidth is designed to be one decade lower from switching frequency to ensure high stability of control signal. Since that converter switching frequency is $25 \mathrm{kHz}$, then the inner loop is design to be $2.5 \mathrm{kHz}$. Outer loop bandwidth is set to be $20 \mathrm{~Hz}$. The controller is design to have a low voltage ripple and fast dynamic response.

\section{RESULTS AND ANALYSIS}

3.1. Design parameter

The proposed circuit is design based on the parameter as in table 1. All parameter are designs based on $[30,31]$ to achieve CCM operation of the bridgeless SEPIC converter. Simulation work using MATLAB/Simulink on the proposed converter is being done based on the controller and design parameter.

Table 1: Design Parameter of the Circuit

\begin{tabular}{cc}
\hline Parameters & Values \\
\hline Line Frequency, $f_{l}$ & $50 \mathrm{~Hz}$ \\
Switching Frequency, $f_{s}$ & $25 \mathrm{kHz}$ \\
Input Voltage, $V_{\text {in }}$ & $120 \mathrm{Vrms}$ \\
Input inductor, $L_{1}, L_{2}$ & $25.5 \mathrm{mH}$ \\
Intermediate inductor, $L_{3} \& L_{4}$ & $12.3 \mathrm{mH}$ \\
Intermediate Capacitor, $C_{1} \& C_{2}$ & $2 \mathrm{uF}$ \\
Output Capacitor, $C_{3}$ & $480 \mathrm{uF}$ \\
\hline
\end{tabular}

Int J Pow Elec \& Dri Syst, Vol. 10, No. 3, Sep 2019 : 1427 - 1436 


\begin{tabular}{cc}
\hline Output Resistor, R & $34 \Omega$ \\
Output Voltage, $V_{o}$ & $100 \mathrm{~V} \mathrm{dc}$ \\
Power Output, $P_{o}$ & $300 \mathrm{~W}$ \\
\hline
\end{tabular}

\subsection{Simulation result}

Figure 6 shows the input current and input voltage of proposed converter. Both input current and input voltage are in phase and power factor of the converter is 0.98 . This signify that the proposed converter work perfectly as PFC. Figure 7, the input current operates in CCM. It successfully achieves theory of CCM by using the proposed average current mode control. The output voltage shown in Figure 8 achieve the target with 5\% voltage ripple. The current output of proposed converter is shown in Figure 9 with $5 \%$ current ripple. The average output power of the bridgeless SEPIC converter successfully achieves $300 \mathrm{~W}$ as anticipate.

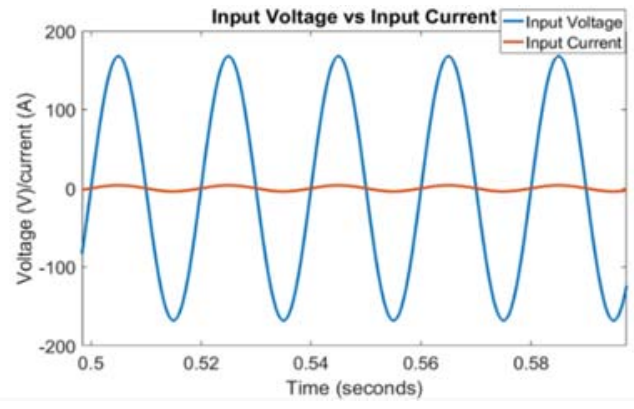

Figure 6. Input voltage and input current

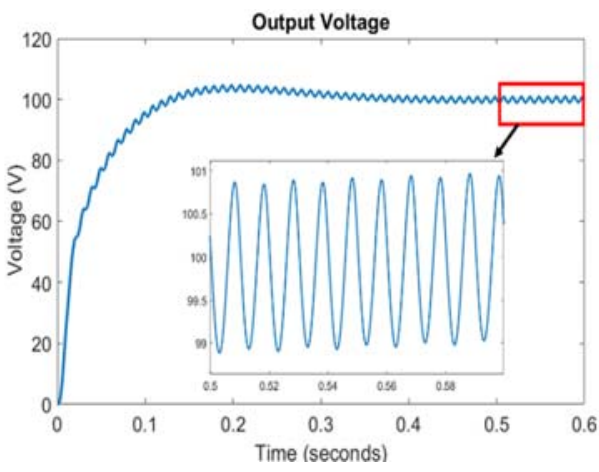

Figure 8. Output voltage

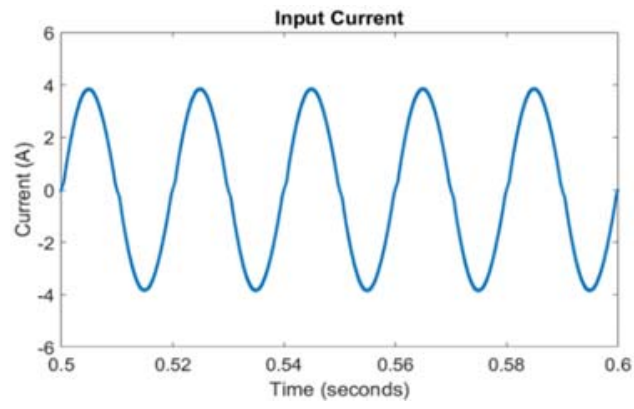

Figure 7. Input current (iL1)

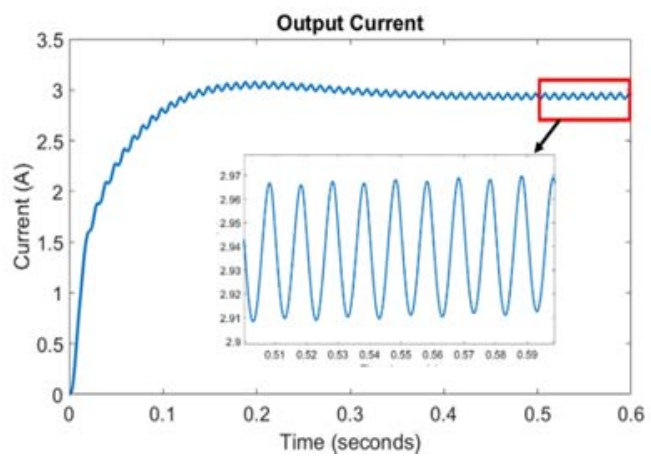

Figure 9. Output current

\subsection{Preliminary hardware result}

Based on simulation, the prototype of bridgeless SEPIC converter have been implement to verify the operation of converter in CCM. DS1104 digital signal processing and control engineering (dSPACE) controller board and basys3 field programmable gate array (FPGA) are used as controller interface to perform ACMC. dSPACE Controldesk control and sense the input voltage, input current and output voltage as proposed. However, dSPACE restricted on lower sampling time with make the accuracy and precision of prototype defer from simulation. By combining dSPACE and FPGA 35us sampling time are achieved. The preliminary result for input current, input voltage, output current and output voltage are shown in Figure 10. 


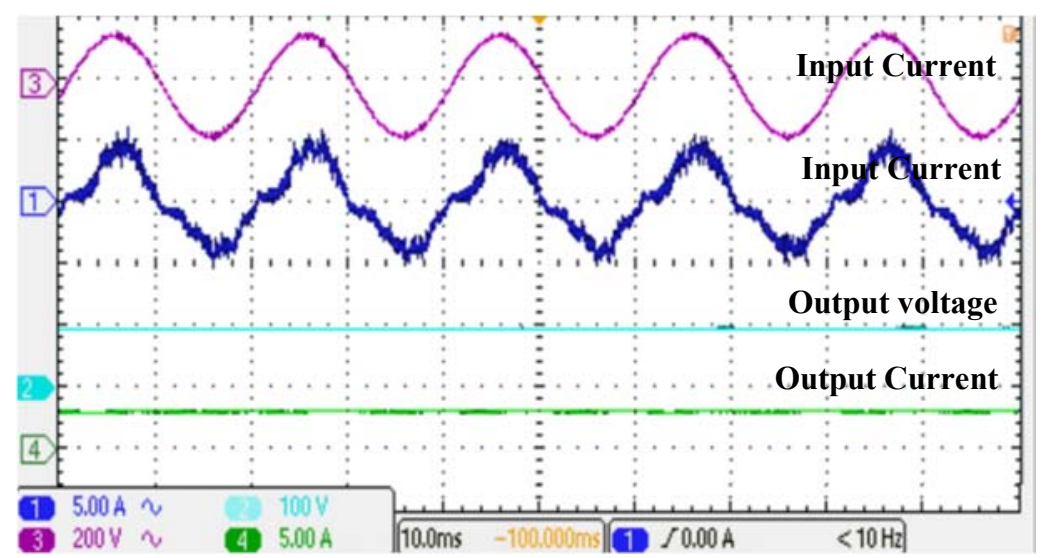

Figure 10. Input voltage, Input current, Output voltage and Output current

The experiment result shows that, the input current and input voltage are in phase and its shape are nearly sinusoidal as simulation result. The output current and voltage ripple is about $5 \%$ same as simulation but slightly have some loss in the hardware test. More detail analysis of the waveform and efficiency performance of the bridgeless SEPIC converter will be discussed in future work.

\section{CONCLUSION}

In this paper, an average current mode controller for asingle phase PFC bridgeless SEPIC converter operated in CCM is proposed and verifed through simulation studies in MATLAB/Simulink. It is shown that the controller is successfully achieved unity power factor for a medium power application (300 W). A prototype of converter is built to verify the performance of theoretical design. Based on the preliminary result, the input current and voltage show a good agreement with 0.92 power factor while at the output side, a smooth dc current and dc voltage with approximately $5 \%$ ripple are obtained.

\section{ACKNOWLEDGEMENTS}

This work was supported by Research University Grant (Q.J130000.2423.15J40 and Q.J130000.2423.14J34) provided by Universiti Teknologi Malaysia.

\section{REFERENCES}

[1] D. W. Hart., Power Electronics. McGraw-Hill Companies, Inc, New York, 2011.

[2] C. Chan and K. Chau., "Power electronics challenges in electric vehicles," International Conference on Industrial Electronics, Control, and Instrumentation, Proceedings of the IECON'93, pp. 701-706, 1993.

[3] D. Borgonovo, Jose, P. Remor, Ivo Barbi \& Arnaldo J. Perin., "A self-controlled power factor correction singlephase boost pre-regulator," IEEE 36th Power Electronics Specialists Conference, pp. 2351-2357, Jun 2005.

[4] T. M. Undeland, W. P. Robbins \& N. Mohan., Power Electronics Converters, Applications and Design. Wiley, New York, NY, USA, 2003

[5] Sidharth Sabyasachi and Pratyusha Mohanty, "Stability analysis of SEPIC converter using matlab/Simulink," International Journal of Electrical, Electronics and Data Communication, vol. 2(6), Jun 2014.

[6] H. Y. Kanaan and K. Al-Haddad., "Small-signal averaged model and carrier-based linear control of a SEPIC-type Power Factor Correction circuit," in Telecommunications Energy Conference, INTELEC, IEEE 30th International, pp. 1-6, 2008.

[7] Jae-Won Yang and Hyun-Lark Do., "Bridgeless SEPIC Converter with a Ripple-Free Input Current," IEEE Transactions on Power Electronics, vol. 28(7), Jul 2013.

[8] Mohammad Mahdavi and Hosein Farzanehfard., "Bridgeless SEPIC PFC Rectifier with Reduced Components and Conduction Losses," IEEE Transactions on Industrial Electronics, vol. 58(9), Sep 2011.

[9] Sahid, M. R and Yatim, A. H M., "Modeling and simulation of a new Bridgeless SEPIC power factor correction circuit," Simulation Modelling Practice and Theory, vol. 19, pp. 596-611, 2011.

[10] Sahid, M. R, A. H. M. Yatim, \& Taufik Taufik., "A new AC-DC converter using bridgeless SEPIC," IECON 201036th Annual Conference on IEEE Industrial Electronics Society, 2010.

Int J Pow Elec \& Dri Syst, Vol. 10, No. 3, Sep 2019 : 1427 - 1436 
[11] Esam H. Ismail., "Bridgeless SEPIC rectifier with unity Power factor and reduced conduction losses," IEEE Transactions on Industrial Electronics, vol. 56(4), Apr 2009.

[12] A. J. Sabzali, E. H. Ismail, M. a. Al-Saffar \& A. a. Fardoun., "New bridgeless DCM Sepic and Cuk PFC rectifiers with low conduction and switching losses," IEEE Transactions on Industry Applications, vol. 47(2), pp. 873-881, 2011.

[13] Lee, Sin-Woo, and Hyun-Lark Do., "Efficient bridgeless PFC converter with reduced voltage stress," International Journal of Circuit Theory and Applications, 2015.

[14] Singh, S. Singh, B \& Bhuvaneswari, G., "Improved power quality SMPS for computers using bridgeless PFC converter at front end," IEEE 6th India International Conference on Power Electronics (IICPE) 2014, pp. 1-6, 2014.

[15] Gegner, Joel P and C. Q. Lee. "Linear peak current mode control: a simple active power factor correction control technique for continuous conduction mode." Power Electronics Specialists Conference, 1996. PESC'96 Record., 27th Annual IEEE, vol. 1, 1996.

[16] O. Garcia and J. A. Cobos., "Single Phase Power Factor Correction: A Survey," IEEE Transactions on Power Electronics, vol. 18(3), pp. 749-755, May 2003.

[17] Kim, JW. Choi, SM \& Kim, KT., "Variable On-time Control of the Critical Conduction Mode Boost Power Factor Correction Converter to Improve Zero-crossing Distortion," 2005 Int Conf Power Electron Drives Syst, vol. 2, pp. 1542-6, 2005.

[18] Rossetto, L., G. Spiazzi, \& P. Tenti., "Control techniques for power factor correction converters," proc. PEMC, vol. 94(9), 1994

[19] H. Wei and I. Batarseh., "Comparison of basic converter topologies for power factor correction," IEEE Proceedings Southeastcon, pp. 348-353, 1998.

[20] Singh, B and Anand, A., "Power Factor Correction in Modified SEPIC Fed Switched Reluctance Motor Drives," In: IEEE Transactions on Industry Applications, vol. 54(5), pp. 4494-4505, 2018.

[21] Ryan, RT. Hayes, JG. Morrison, RJ \& Hogan, DN., "Improved Zero-Crossing Distortion of A BoundaryConduction-Mode Boost Converter with Digital Average-Current-Mode Control,", 2018 IEEE Appl Power Electron Conf Expo, pp. 1846-5, 2018.

[22] L. H. Dixon, Average current mode control of switching power supplied. Unitrode power supply Design Seminar Handbook, 1990.

[23] C. Zhou and M. Jovanovic., "Design trade-offs in continuous current-mode controlled boost power-factor correction circuits," in Proc. High-Frequency Power Conversion Conf, pp. 209-219, 1992.

[24] Yong Zhang; Weisheng Xu \& Youling Yu, "The PFC with Average Current-Mode and Voltage Fuzzy Controller for the Output Voltage," Proceedings of Intelligent Information Technology Application, 2008. IITA '08. Second International Symposium, 20-22 Dec 2008.

[25] V. Eng, U. Pinsopon, and C. Bunlaksananusorn, "Modeling of a SEPIC converter operating in continuous conduction mode," in Electrical Engineering/Electronics, Computer, Telecommunications and Information Technology, 2009. ECTI-CON 2009. 6th International Conference on, vol. 1, pp. 136-139, 2009.

[26] H. Y. Kanaan and K. Al-Haddad, "Small-signal averaged model and carrier-based linear control of a SEPIC-type Power Factor Correction circuit," in Telecommunications Energy Conference, INTELEC, IEEE 30th International, 2008, pp. 1-6.

[27] Middlebrook, RD and Cuk, S., "A general unified approach to modelling switching-converter power stages," InPower Electronics Specialists Conference, pp. 18-34, 1976.

[28] Krein, P.T., Elements of Power Electronics (1st ed.). Oxford University Press, New York, 1998

[29] M. V. M. Ewerling and C. H. I. Font., "Single-stage AC/DC converter based on SEPIC topology operating in continuous conduction mode," 2017 Brazilian Power Electronics Conference (COBEP), pp. 1-6, Juiz de Fora, 2017.

[30] Sebastian, J., J. A. Cobos., J. M. Lopera, \& U. Uceda., "The determination of the boundaries between continuous and discontinuous conduction modes in PWM DC-to-DC converters used as power factor preregulators." IEEE transactions on power electronics, vol. 10(5), pp. 574-582, 1995.

[31] Kong, Pui Yee, J. A. Aziz, M. R. Sahid, \& Low Wen Yao., "A bridgeless PFC converter for on-board battery charger," In Energy Conversion (CENCON), 2014 IEEE Conference on, pp. 383-388, 2014. 


\section{BIOGRAPHIES OF AUTHORS}

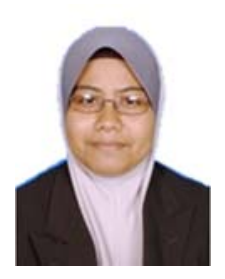

Nor Akmal Rai received her B.E (Electrical) degree from Universiti Sains Malaysia (USM) in 2011 and the Master in Electrical Engineering (Power) from Universiti Teknologi Malaysia (UTM) in 2015. She is currently pursuing Ph.D degree at Faculty of Electrical Engineering, Universiti Teknologi Malaysia (UTM), Malaysia. Her research interests include power factor correction, power electronic and electric vehicle charger.

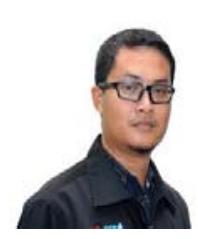

Mohd Junaidi Abdul Aziz received his B.S. and M.S. degrees in Electrical Engineering from the Universiti Teknologi Malaysia (UTM), Kuala Lumpur, Malaysia, in 2000 and 2002, respectively; and his Ph.D. in Electrical Engineering from The University of Nottingham, Nottingham, England, UK, in 2008. Since 2008, he has been with the Faculty of Electrical Engineering, UTM, where he is presently an associate professor. His current research interests include power electronics and electric vehicles with a special focus on battery management systems.

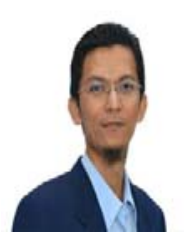

Mohd Rodhi Sahid received his Degree in Bachelor of Electrical Engineering, Master in Electrical Engineering (Power) and Doctor of Philosophy (Electrical) from Universiti Teknologi in year 2000, 2003 and 2012, respectively. He has been with the Faculty of Electrical Engineering, UTM, where he is presently a Senior Lecturer. His current research interests include area of interest includes power factor correction, DC-DC converters and power electronic.

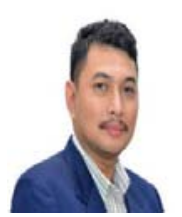

Shahrin Md Ayob received his Degree in Bachelor of Electrical Engineering, Master in Electrical Engineering (Power) and Doctor of Philosophy (Electrical) from Universiti Teknologi in year 2000, 2003 and 2010, respectively. Currently he is an associate professor in School of Electrical Engineereing, Faculty of Engineering, Universiti Teknologi Malaysia. He was a chair of Power Electronics Society Malaysia Chapter in year 2017-2018. His current research is in multilevel topology, fuzzy control and electric vehicle. 